\title{
Proficiency of eye drop instillation in postoperative cataract patients in Ghana
}

This article was published in the following Dove Press journal:

Clinical Ophthalmology

25 October 2013

Number of times this article has been viewed

Yang Liu'

Amanda Murdoch ${ }^{2}$

Ken Bassett ${ }^{3}$

Shafik Dharamsi ${ }^{4}$

'Faculty of Land and Food Systems, University of British Columbia, Vancouver, BC, Canada; '2Queen's School of Medicine, Queen's University, Kingston, ON, Canada; ${ }^{3}$ Departments of Family Practice and Ophthalmology and Visual Sciences, University of British Columbia, Vancouver, BC, Canada; ${ }^{4}$ Department of Family Practice, University of British Columbia, Vancouver, BC, Canada
Correspondence: Amanda Murdoch Queen's University Undergraduate Medical Education Office, Undergraduate Medical Education, Queen's University, 80 Barrie Street, Kingston, ON,

K7L 3N6, Canada

Tel + I 6139857384

Email amurdoch@qmed.ca
Objective: The purpose of the study was to evaluate the efficacy of postoperative instruction on proficiency of eye drop instillation following cataract surgery, and to determine whether such proficiency correlates with the prevalence and/or duration of irritation and pain experienced in operated eyes.

Design: This was a prospective, nonrandomized control trial with an educational intervention conducted via a single eye clinic in Accra, Ghana.

Participants: The 218 subjects who completed the study were postoperative cataract surgery patients whose surgery had been funded by the nongovernmental organization Unite for Sight.

Methods: Patients were evaluated on their ability to administer eye drops correctly on their first attempt on postoperative day one. If unsuccessful, patients were given an educational session that consisted of verbal instructions and an educational video. Both groups (successful and unsuccessful) on the first postoperative day were tested again for proficiency on postoperative day 30. The baseline group was evaluated only on postoperative day 30 and consisted of 36 patients.

Results: Of the 133 patients who received the educational session on eye drop instillation, $112(84 \%)$ exhibited proficiency on postoperative day 30 as compared with 29 of 49 patients $(59 \%)$ who did not receive the intervention. Additionally, there were fewer reports of pain and irritation following cataract extraction in the patients who received the educational session.

Conclusion: This study supports the efficacy of patient education in improving proficiency in eye drop instillation and in reducing pain and irritation following cataract extraction surgeries.

Keywords: cataract extraction, ophthalmic solutions, postoperative complications, patient education

\section{Introduction}

Cataracts are a leading cause of treatable blindness that affects millions of people worldwide, particularly in developing countries. ${ }^{1,2}$ This burden of disease represents a significant global health problem. Researchers have studied postoperative complications and interventions to prevent them. ${ }^{3,4}$ Postoperative care following cataract extractions commonly involves the administration of antibiotic and anti-inflammatory steroid-based eye drops. This can help to prevent infection and cystoid macular edema, as well as controlling intraocular inflammation and pain. ${ }^{5,6}$ Topical antibiotics are the international standard of care following cataract surgery. ${ }^{7,8}$ Medicated eye drops containing dexamethasone, neomycin sulfate, and polymyxin B sulfate, such as the treatment used in this study, are commonly used internationally to control 
postoperative inflammation and prevent infection. ${ }^{9}$ Improper administration of postoperative eye drops can increase the risk of ocular infection. ${ }^{10}$ There is also a potential risk of side effects from excess medication draining into the nasolacrimal system. ${ }^{11,12}$

Education regarding appropriate eye drop utilization is particularly important in areas with low literacy rates and limited formal education. Kholdebarin et $\mathrm{al}^{13}$ have reported that improper administration techniques were associated with the patients' formal education limited to elementary school. Muir et $\mathrm{al}^{14}$ confirmed that subjects demonstrating low literacy levels were less adherent to their glaucoma medications than those with higher literacy levels. Literacy levels in Ghana are generally low (57.9\%), especially in rural areas, where literacy is defined as the ability to read and write among individuals aged 15 years and older. ${ }^{15}$

The purpose of this study was to evaluate the efficacy of postoperative patient instruction on the proficiency of eye drop instillation following cataract surgery, and to determine whether such proficiency correlates with the prevalence and/or duration of irritation and pain experienced by patients postoperatively. This study focused on a poorly literate, nonurban population. All subjects in the study lived in rural Ghanaian villages and received manual small incision cataract surgery (SICS) at the Crystal Eye Clinic in Accra, Ghana. The investigators in this study have not been able to identify studies analyzing the efficacy of eye drop instillation in postoperative cataract patients, though research has been conducted regarding patient administration of glaucoma medication. ${ }^{13,16,17}$

\section{Materials and methods Participants}

The research protocol used in this study followed the principles delineated by the Declaration of Helsinki and was approved by the Clinical Research Ethics Board of the University of British Columbia, Unite For Sight (UFS), and the Crystal Eye Clinic.

This study was conducted in the Crystal Eye Clinic in Accra, Ghana. Eligible study participants were postoperative cataract surgery patients over the age of 19 years. The cataract extraction technique used was SICS. Subjects were outreach patients from a village, slum, or refugee camp, whose cataract surgery was sponsored by UFS. UFS fully covered all medical expenses associated with the surgery, overnight lodging, and transportation, if needed. Consent was obtained following a detailed explanation of the study by a native speaker in the patient's language of choice
(Twi, Fante, Ewe, Dangme, Ga, or Hausa). Written consent was requested, but because of challenges with literacy, verbal consent was also accepted. The study period occurred from August 2010 to November 2010.

218 subjects completed the study; three additional subjects were present for postoperative day one (POD \#1), but did not return for postoperative day 30 (POD \#30); their data were not included in the analysis.

\section{Design}

All participants enrolled in the study received treatment as usual for cataract extraction, including a standard postoperative educational session from the clinic staff. When self-administration of eye drops was unmanageable, relatives who accompanied the patients were given the eye-drop administration tutorial. The educational session included the importance of hand washing and avoiding touching the tip of the bottle to any surfaces, as well as demonstrating the appropriate eye drop application technique. This tutorial takes 90 to 120 seconds. For the purposes of the study, proficient eye drop administration was defined by the three criteria outlined by Stone et al: ${ }^{16} 1$ ) the drops should touch and be retained on the ocular surface; 2) the tip of the bottle should not touch the eye or ocular adnexa; and 3) only one eye drop should be applied.

The study population was divided into three groups, A, B, and $\mathrm{C}$ (Figure 1). Group A served as the control group; subjects received the standard clinic postoperative instructions and were only assessed for eye drop proficiency on POD \#30. Group A subjects had their POD \#30 check-up within the study period, but received their UFS-sponsored surgery prior to the study period. Group A patients thus consented to the study at their POD \#30 check-up.

Subjects in Group B also received the standard clinic postoperative instructions, and their attempts to administer eye drops were observed on POD \#1 by the researcher. If the drops were administered correctly on the first attempt, the participants remained in Group B. Participants who were unable to administer the single eye drop correctly on their first attempt on POD \#1 were classified into Group C. The participants were also observed for proficiency when they returned for their POD \#30 follow-up visits.

When the Group C participants incorrectly administered eye drops on the first attempt, they received additional verbal instructions on correct eye drop administration in the language of their choice. They were also shown a video of a clinic staff member demonstrating the proper technique on a laptop or camera. When the patient's visual acuity did not permit 


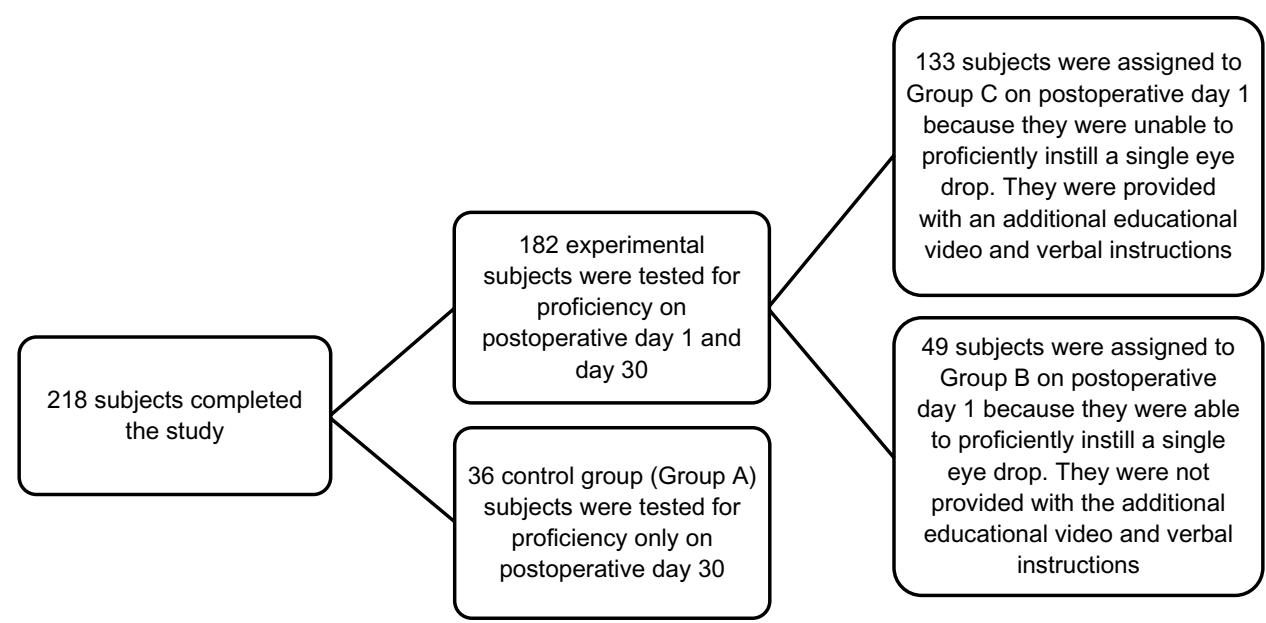

Figure I Flow chart illustrating study design and group allotment.

adequate observation of the video, a researcher demonstrated the proper eye drop administration technique with normal saline. This educational session was approximately 5 minutes in duration. The session was feasible to apply at the Crystal Eye Clinic, both in terms of time and materials, since UFS volunteers generally carry their cameras or laptops with them during clinic shifts and could load the educational video onto these devices. The participants were also observed for proficiency when they returned for their POD \#30 follow-up visits.

\section{Video script}

The audio script that accompanied the video was as follows:

This video will demonstrate how to apply eye drops properly. Pull down your lower eyelid to form a pouch that the drop should fall into. Apply a single drop to the eye that received surgery and close both eyes for 30 seconds. Only one drop is needed; adding more drops will not make the medication work better, it will only waste the medication. Squeezing the bottle harder will make drops come out faster so be aware of how much force is needed to get only one drop out of the bottle. Make sure that the tip of the bottle doesn't touch your eyelashes, eyelids, or any other part of your eye.

\section{Protocol}

Participant eye drop administration proficiency was assessed through the following protocol. Subjects were provided with $15 \mathrm{~mL}$ bottles of the topical ophthalmic solution Dexatrol (Egyptian International Pharmaceutical Industries Company [EPICO], Tenth of Ramadan City, Egypt), containing dexamethasone, neomycin sulfate, and polymyxin B sulphate. ${ }^{18}$ Subjects were instructed to wash their hands and to apply one drop of Dexatrol into the operated eye. This process was observed by the researcher and interpreter.

Subsequently, subjects also completed a verbal survey regarding their understanding of eye drop administration instructions, their perceived proficiency in eye drop administration, any factors that would limit their ability to administer eye drops proficiently, and their history of eye drop usage.

\section{Statistical analysis}

Video recordings of patients administering eye drops were independently scored by two researchers using the three criteria for proficiency. No sample size estimate was performed. All data from the surveys and video scoring were analyzed using analysis of variance (ANOVA) $(\alpha=0.05)$.

\section{Results}

On POD \#1, researchers found that $26.92 \%$ (49/182) of Groups $\mathrm{B}$ and $\mathrm{C}$ subjects were able to administer drops proficiently. On POD \#30, Group B subjects who demonstrated 100\% proficiency levels on POD \#1 demonstrated a decrease in proficiency levels to $59.18 \%$ (29/49). Group C subjects who demonstrated $0 \%$ proficiency levels on POD \#1 and were provided with an educational intervention, demonstrated proficiency levels of $84.21 \%(112 / 133)$ on POD \#30. The baseline group (Group A) demonstrated a proficiency level of $8.33 \%$ (3/36) on POD \#30.

There was also a distinct difference in the reported proficiency of eye drop instillation when compared with observed proficiency amongst study participants (Table 1 and Figure 2). The measured POD \#30 eye drop instillation proficiency ranged from $8 \%-84 \%$ across the three study groups, yet participants from all three groups self-reported their proficiency from $87 \%-91 \%$. There was no statistical 
Table I Postoperative day 30 (POD \#30) proficiency (measured versus reported)

\begin{tabular}{|c|c|c|c|c|}
\hline & Group A & Group B & Group C & $P$-value \\
\hline $\begin{array}{l}\text { Measured: eye drop instillation proficiency } \\
\text { on postoperative day } \# 30\end{array}$ & $8.33 \%(3 / 36)$ & $59.18 \%(29 / 49)$ & $84.21 \%(1 / 1 / / 33)$ & 0.002 \\
\hline $\begin{array}{l}\text { Reported: percentage of subjects who do not have } \\
\text { trouble applying eye drops }\end{array}$ & $91.67 \%(33 / 36)$ & $87.76 \%(43 / 49)$ & $91.73 \%(122 / 133)$ & 0.17 \\
\hline $\begin{array}{l}\text { Reported: percentage of subjects who do not miss their } \\
\text { eye when applying eye drops }\end{array}$ & $69.44 \%(25 / 36)$ & $63.27 \%(31 / 49)$ & $72.93 \%(97 / / 33)$ & 0.18 \\
\hline $\begin{array}{l}\text { Reported: percentage of subjects who do not touch the } \\
\text { eye with the bottle tip }\end{array}$ & $61.11 \%(22 / 36)$ & $81.63 \%(40 / 49)$ & $72.18 \%(96 / 133)$ & 0.10 \\
\hline
\end{tabular}

difference between the three groups in terms of the percentage of patients who reported "no trouble applying eye drops", "did not miss their eye when applying eye drops", and "did not touch their eye with the bottle tip".

There was a statistically significant difference between the reports of pain and irritation following surgery between the three groups (Table 2 and Figure 3). A significant number of Group A subjects reported feeling pain or irritation on POD \#30 compared with Group B and C subjects. This same pattern was observed in subjects who did not experience pain or irritation on POD \#30, but had experienced pain or irritation between POD \#1 and POD \#30. Group A subjects also reported a higher mean duration of pain or irritation: 3.53 days for Group A compared to 1.20 days and 0.77 days for Groups $\mathrm{B}$ and $\mathrm{C}$, respectively. As patients were seen by researchers only on POD \#1 and POD \#30, any physical signs of irritation such as erythema and edema were not measured. Postoperative irritation was assessed by patient self-report.

Data regarding proficiency of administration were separated into the three performance criteria in order to analyze the areas of strength and weakness for the majority of subjects. Among the subjects, 48.17\% (105/218) touched the tip of the bottle to the eye or ocular adnexa. The inability to instill only one drop was observed to be the primary problem: $56.88 \%(124 / 218)$ of subjects were unable to instill only a single drop of the medication.

\section{Discussion}

As mentioned, the investigators did not find studies that focused on eye drop administration in postoperative cataract patients to compare with this data. Hence, studies on eye drop administration among glaucoma patients were used for comparison. Stone et $\mathrm{al}^{16}$ evaluated the proficiency levels

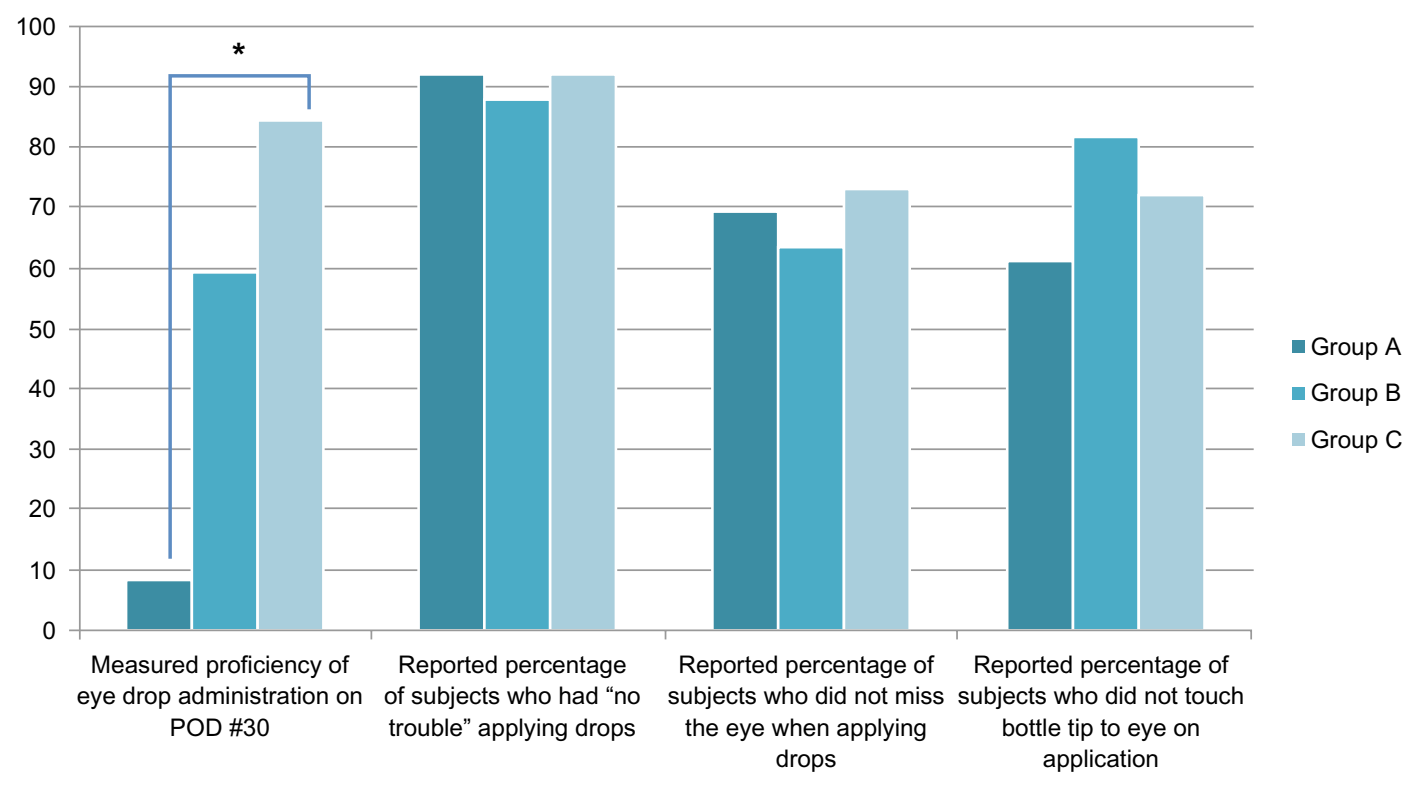

Figure 2 Postoperative day 30 (POD \#30) proficiency (measured versus reported).

Note: The differences in true eye drop instillation proficiency and patient-perceived proficiency via direct observation of technique and self-reporting, respectively. There was a significant difference in the measured proficiency of eye drop instillation between Group A and Group C, $(P$-value: 0.002$)$. There was no statistically or clinically significant difference in patient perception of eye drop instillation proficiency. *indicates statistical significance with a $P$-value $<0.05$. 
Table 2 Questionnaire responses regarding postoperative pain and irritation

\begin{tabular}{|c|c|c|c|c|}
\hline & Group A & Group B & Group C & $P$-value \\
\hline Currently experiencing pain in eye that received cataract surgery & $16.67 \%(6 / 36)$ & $0 \%(0 / 49)$ & $1.50 \%(2 / 133)$ & 0.002 \\
\hline Currently experiencing irritation in eye that received cataract surgery & $16.67 \%(6 / 36)$ & $2.04 \%(1 / 49)$ & $1.50 \%(2 / 133)$ & 0.0015 \\
\hline $\begin{array}{l}\text { Experienced pain in eye that received cataract surgery } \\
\text { from POD } \# I \text { to POD } \# 30\end{array}$ & $44.44 \%(16 / 36)$ & $12.24 \%(6 / 49)$ & $8.27 \%(11 / 133)$ & 0.00186 \\
\hline $\begin{array}{l}\text { Experienced irritation in eye that received cataract surgery } \\
\text { from POD \#I to POD \#30 }\end{array}$ & $41.67 \%(15 / 36)$ & $18.37 \%(9 / 49)$ & $12.78 \%(17 / 133)$ & 0.0036 \\
\hline Duration of pain or irritation, days (standard deviation) & $3.53(4.02)$ & $1.20(2.18)$ & $0.77(1.62)$ & 0.0016 \\
\hline
\end{tabular}

Note: The number of responses over the number of subjects is shown in parentheses.

Abbreviations: POD \#I, postoperative day one; POD \#30, postoperative day 30.

of patients who had used eye drops for at least 6 months. Proficiency levels were measured based on the same criteria used in this study. These researchers found that participants had significantly low success rates: only $20 \%-30 \%$ of subjects were capable of administering a single eye drop without touching their eye or adnexa. This agrees with the data from this study for POD \#1 (Group B and Group C combined), in which only $26.92 \%$ of subjects were able to administer eye drops proficiently.

On POD \#30, the investigators observed that the percentage of subjects who were able to instill eye drops that touched and held on the ocular surface was $63.76 \%(139 / 218)$. Gupta et a $\mathrm{l}^{19}$ measured the proficiency of patients who had used glaucoma medication for 6 months with the same criteria and observed a similar percentage: $68.6 \%(48 / 70)$. Kass et $\mathrm{al}^{20}$ and Hosoda et $\mathrm{al}^{21}$ noted incidences of $49.6 \%$ and $54.9 \%$, respectively, of bottle-tip contamination as a result of contact with ocular or periocular tissues during their observations of eye drop instillation in glaucoma patients. In our study, $48.17 \%(105 / 218)$ of subjects touched the tip of the bottle to their eye or ocular adnexa.

Patients from the Stone et al ${ }^{16}$ study also self-reported relatively high performance concerning their ability to administer the eye drops. Specifically, of the 139 patients, 129 (92.8\%) reported no problem administering their eye drops, 86 (61.9\%) believed that they never missed their eye when administering the eye drops, and 110 (79.2\%) believed that they never touched their eye or adnexa with the bottle tip. Similarly, in this study, there was no statistically significant difference between the three groups in terms of their responses regarding problems administering eye drops, missing the eye when administering the drops, and touching the bottle tip to the eye. On POD \#30 of this postoperative cataract medication study, 198 (90.83\%) subjects reported no problem administering their eye drops, 153 (70.18\%) believed that they never missed their eye when administering

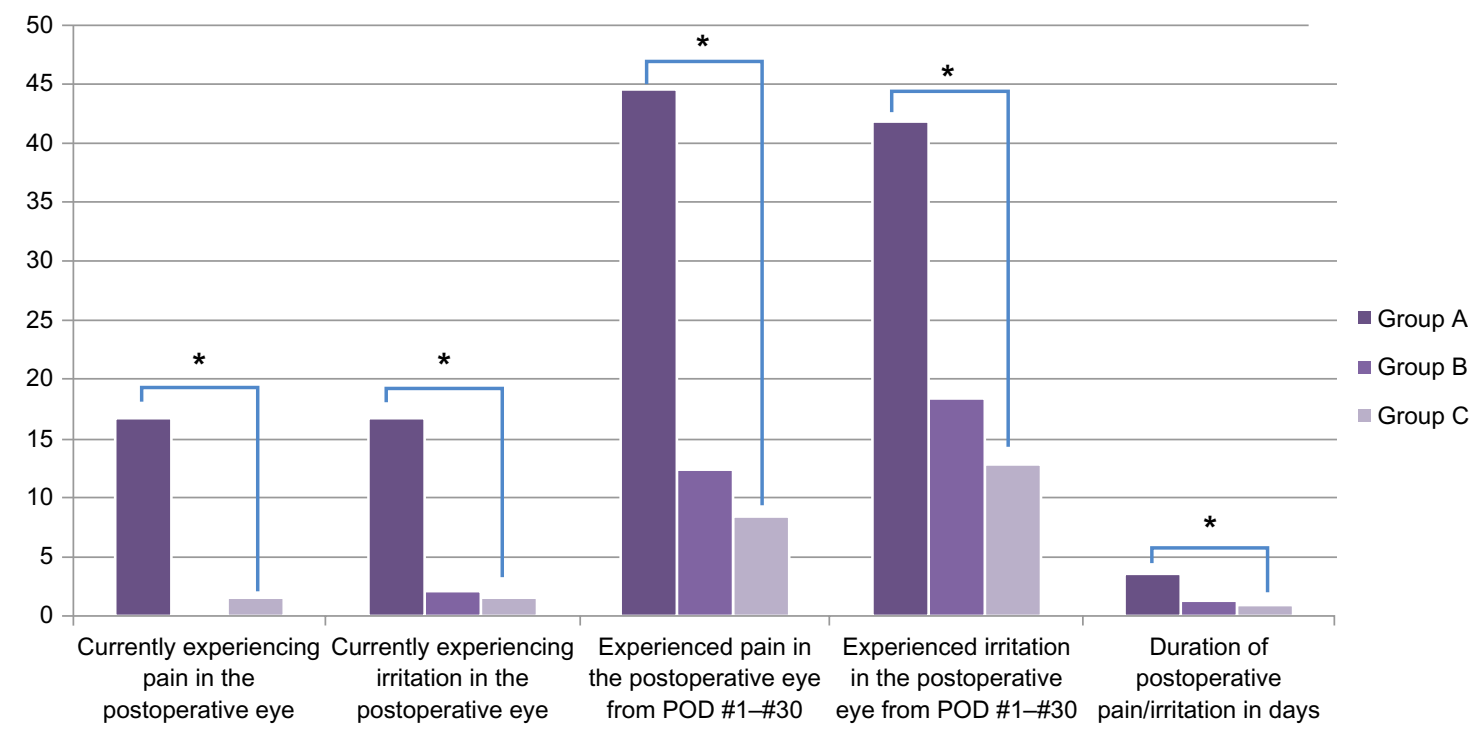

Figure 3 Questionnaire responses regarding postoperative pain and irritation.

Notes: Subject questionnaire responses collected on POD \#30 concerning pain and irritation that were experienced in the time following the surgical procedure. Subjects were asked about symptoms at the time the questionnaire, as well as in weeks from the procedure and the questionnaire. A one-way analysis of variance of the three groups found a significant difference between the groups across all five pain and irritation categories. *indicates statistical significance with a $P$-value $<0.05$.

Abbreviations: POD \#I, postoperative day one; POD \#30, postoperative day 30. 
the drops, and $158(72.48 \%)$ believed they never touched their eye or adnexa with the bottle tip. Patient perceptions about their performance do not agree with objective observations, as patients may want to please the researcher, or do not feel comfortable admitting that they have problems applying the medication. They also may not have been aware of their level of medication administration skill. ${ }^{22}$

Of the three criteria used to evaluate proficiency, it was observed that the inability to apply only one drop was the primary problem. For future studies, it may be beneficial to identify why this was the case, and to determine whether this was a result of subjects missing the eye completely, or administering more than one drop. Targeting this aspect of drop administration in educational interventions may be beneficial in reducing waste. Although patients who receive surgery sponsored by UFS have their postoperative medication costs covered, patients who do not need surgery but require eye drops must pay a small fee for the eye drops and may not be aware that with each drop wasted, they may be spending more money than necessary. ${ }^{17}$ It would be worthwhile to also document whether patients touch the bottle tip with their finger or any other surfaces that could cause contamination. Problems with hygiene and sanitation in the slums and refugee camps, where many of the patients lived, make infectious diseases prevalent.

The mean age ( \pm standard deviation) of the subjects was $70.6 \pm 5.1$ years. Subjects who reported problems with eye drop administration indicated that this was a result of arthritis, shaking hands, and poor vision in the eye that was not operated on. A limitation in comparing this study's results with previously published studies on proficiency of eye drop administration is that all of the studies cited took place in North America, whereas this study focuses on subjects from impoverished communities in rural Ghana. We did not collect data on participant literacy rates, though the great majority of our participants were not literate or had low literacy, as nearly all gave verbal rather than written consent. As such, we cannot correlate patient literacy rates with eye drop instillation proficiency. Future studies could examine the effect of literacy on the proficiency of eye drop instillation in a more diverse participant population.

Another limitation to this study is the fact that the observation of a single session of eye drop instillation for only one eye may have resulted in an observation that differs from the subject's usual performance. Additionally, the presence of observers may also have influenced the subject's performance. ${ }^{16}$ A limitation to the study protocol was the observation stage of assessment. The initial protocol was to record patient eye drop administration via video camera, as per Stone et al. ${ }^{16}$ Upon arrival in the clinic and rural communities in Ghana, it was determined that this approach was not feasible, and patient evaluation was done by the researcher in real time.

In conclusion, the results demonstrated that subjects who received the educational session had higher proficiency levels and fewer reports of pain and irritation, as well as shorter durations of pain and irritation, than subjects who did not receive the tutorial. The study also confirmed that patient perceptions about their proficiency in eye drop administration do not agree with objective observations. Providing the educational video with the verbal message in the patient's primary language to each postoperative cataract patient would be beneficial.

\section{Acknowledgments}

We would like to thank Tiffany Shiau for her help in collecting data and reviewing the paper. We would also like to acknowledge Dr James Clarke, Jennifer Staple-Clark, Laura Reynolds, Rachel Turkel, Jo-Ann Osei-Twum, Dr Jerry Spiegel, and Dr Annalee Yassi for their contributions to the design of this study. We would like to thank Jerome Yevugah for helping us create the educational video, Dr Ernest Awiti for his technical assistance, and John Austin Agbeletorme for acting as our interpreter.

\section{Disclosure}

The authors have no conflicts to declare with regards to financial interests or associations.

\section{References}

1. Rao GN, Khanna R, Payal A. The global burden of cataract. Curr Opin Opthalmol. 2011;22(1):4-9.

2. Tabin G, Chen M, Espandar L. Cataract surgery for the developing world. Curr Opin Opthalmol. 2008;19(1):55-59.

3. Powe NR, Schein OD, Gieser SC, et al. Synthesis of the literature on visual acuity and complications following cataract extraction with intraocular lens implantation. Arch Ophthalmol. 1994;112(2):239-252.

4. Nordlund ML, Marques DM, Marques FF, Cionni RJ, Osher RH. Techniques for managing common complications of cataract surgery. Curr Opin Ophthalmol. 2003;14(1)1:7-19.

5. Rowen S. Preoperative and postoperative medications used for cataract surgery. Curr Opin Ophthalmol. 1999;10(1):29-35.

6. Laurell CG, Zetterström C. Effects of dexamethasone, diclofenac, or placebo on the inflammatory response after cataract surgery. $\mathrm{Br} J$ Ophthalmol. 2002;86(12):1380-1384.

7. Braich PS, Almeida DR, Hollands S, Coleman MT. Effects of pictograms in educating 3 distinct low-literacy populations on the use of postoperative cataract medication. Can J Ophthalmol. 2011;46(3):276-281.

8. Masket S, Steinert RF, Mah FS. Antibiotics in cataract surgery. Cataract and refractive surgery today. Available from: http://www.crstoday. com/PDF\%20Articles/0308/CRST0308_03.php. Accessed January 10, 2012 
9. Notivol R, Bertin D, Amin D, Whitling A, Kennedy M, Cockrum PC; C-98-64 Study Group. Comparison of topical tobramycin dexamethasone with dexamethasone-neomycin-polymyxin and neomycin-polymyxingramicidin for control of inflammation after cataract surgery: results of a multicenter, prospective, three-arm, randomized, double-masked, controlled, parallel-group study. Clin Ther. 2004;26(8):1274-1285.

10. Geyer O, Bottone EJ, Podos SM, Schumer RA, Asbell PA. Microbial contamination of medications used to treat glaucoma. Br J Ophthalmol. 1995;79(4):376-379.

11. Tsai T, Robin AL, Smith JP. An evaluation of how glaucoma patients use topical medications: a pilot study. Trans Am Ophthalmol Soc. 2007; 105:29-33.

12. Lederer CM Jr, Harold RE. Drop size of commercial glaucoma medications. Am J Opththalmol. 1986;101(6):691-694.

13. Kholdebarin R, Campbell RJ, Jin YP, Buys YM. Multicenter study of compliance and drop administration in glaucoma. Can J Ophthalmol. 2008;43(4):454-461.

14. Muir KW, Santiago-Turla C, Stinnett SS, et al. Health literacy and adherence to glaucoma therapy. Am J Ophthalmol. 2006;142(2): 223-226.

15. Central Intelligence Agency Web Site. The World Factbook. Available from: https://www.cia.gov/library/publications/the-world-factbook/ fields/2103.html. Accessed September 7, 2013.
16. Stone JL, Robin AL, Novack GD, Covert DW, Cagle GD. An objective evaluation of eyedrop instillation in patients with glaucoma. Arch Ophthalmol. 2009;127(6):732-736.

17. Hennessy AL, Katz J, Covert D, Protzko C, Robin AL. Videotaped evaluation of eyedrop instillation in glaucoma patients with visual impairment or moderate to severe visual field loss. Ophthalmology. 2010;117(12):2345-2352.

18. Egyptian International Pharmaceutical Industries CO. Web Site. Dexatrol. Available from: http://www.eipico.com.eg/PRODUCT. ASP?id1=17\&id2=6\%20\&id4=3. Accessed September 7, 2013.

19. Gupta R, Patil B, Shah B, Bali SJ, Mishra SK, Dada T. Evaluating eye drop instillation technique in glaucoma patients. J Glaucoma. 2012;21(3):189-192.

20. Kass MA, Hodapp E, Gordon M, Kolker AE, Goldberg I. Patient administration of eyedrops: observation. Part II. Ann Ophthalmol. 1982;14(9):889-893.

21. Hosoda M, Yamabayashi S, Furuta M, Tsukahara S. Do glaucoma patients use eye drops correctly? J Glaucoma. 1995;4(3):202-206.

22. Kass MA, Meltzer DW, Gordon M, Cooper D, Goldberg J. Compliance with topical pilocarpine treatment. Am J Ophthalmol. 1986;101(5) $515-523$.
Clinical Ophthalmology

\section{Publish your work in this journal}

Clinical Ophthalmology is an international, peer-reviewed journal covering all subspecialties within ophthalmology. Key topics include: Optometry; Visual science; Pharmacology and drug therapy in eye diseases; Basic Sciences; Primary and Secondary eye care; Patien Safety and Quality of Care Improvements. This journal is indexed on

Submit your manuscript here: http://www.dovepress.com/clinical-ophthalmology-journal

\section{Dovepress}

PubMed Central and CAS, and is the official journal of The Society of Clinical Ophthalmology (SCO). The manuscript management system is completely online and includes a very quick and fair peer-review system, which is all easy to use. Visit http://www.dovepress.com/ testimonials.php to read real quotes from published authors. 